Published in final edited form as:

Curr Opin Oncol. 2012 November ; 24(6): 650-654. doi:10.1097/CCO.0b013e328356da72.

\title{
Glucose transporters in cancer metabolism
}

\author{
Kehinde Adekola ${ }^{a, b}$, Steven T. Rosen ${ }^{a, b}$, and Mala Shanmugam ${ }^{a}$ \\ ${ }^{a}$ Robert H. Lurie Comprehensive Cancer Center, Northwestern University Feinberg School of \\ Medicine, Chicago, Illinois, USA \\ ${ }^{\mathrm{b}}$ Division of Hematology/Oncology, Northwestern University Feinberg School of Medicine, \\ Chicago, Illinois, USA
}

\begin{abstract}
Purpose of review-Transformed cells exhibit a high rate of glucose consumption beyond that necessary for ATP synthesis. Glucose aids in the generation of biomass and regulates cellular signaling critical for oncogenic progression. A key rate-limiting step in glucose utilization is the transport of glucose across the plasma membrane. This review will highlight key glucose transporters (GLUTs) and current therapies targeting this class of proteins.
\end{abstract}

Recent findings-GLUTs, enabling the facilitative entry of glucose into a cell, are increasingly found to be deregulated in cancer. Although cancer-specific expression patterns for GLUTs are being identified, comprehensive analyses substantiating a role for individual GLUTs are still required. Studies defining GLUTs as being rate-limiting in specific tumor contexts, the identification of GLUT1 inhibitors via synthetic lethality screens, novel engagement of the insulinresponsive GLUT4 in myeloma and identification of GLUT9 being a urate transporter, are key advances underscoring the need for continued investigation of this large and enigmatic class of proteins.

Summary-Tumor cells exhibit elevated levels of glucose uptake, a phenomenon that has been capitalized upon for the prognostic and diagnostic imaging of a wide range of cancers using radiolabeled glucose analogs. We have, however, not yet been able to target glucose entry in a tumor cell-specific manner for therapy. GLUTs have been identified as rate-limiting in specific tumor contexts. The identification and targeting of tumor-specific GLUTs provide a promising approach to block glucose-regulated metabolism and signaling more comprehensively.

\section{Keywords}

cancer metabolism; glucose transporter; Warburg effect

Correspondence to Mala Shanmugam, Robert H. Lurie Comprehensive Cancer Center, Northwestern University, 303 East Chicago Avenue, Lurie 3-250, Chicago, IL 60611, USA. Tel: +1 312908 5919; fax: +1 312503 0386; mala@ northwestern.edu.

Conflicts of interest

The authors declare no competing financial interests. K.A. has received funding from NCI T32 CA079447. M.S. has received funding from American Cancer Society (Illinois Division \#188679), American Cancer Society Research Scholar grant (RSG-11-254-01CSM) and Wendy Will Case Cancer Fund Foundation (SP0012544). 


\section{INTRODUCTION}

Glucose is a major source of energy in mammalian cells, generating ATP through glycolysis and oxidative phosphorylation. It is also a precursor of amino acids, nucleotide and lipids [1]. The transport of glucose across the plasma membrane into the cytosol is a rate-limiting step in glucose metabolism and is mediated by a family of glucose transporters (GLUTs) [24]. Facilitative GLUTs enable the ATP-independent, bi-directional transportation of glucose across a hydrophobic cell membrane, down its concentration gradient $[2,3]$. This review will aim to outline the GLUT gene family, their functions and role in cancer metabolism and highlight therapeutic interventions that target them.

\section{GLUCOSE METABOLISM AND GLUCOSE TRANSPORTATION IN CANCER}

One of the hallmarks of cancer cell development is the increased dependence on glucose to fuel aerobic glycolysis for the increased production of cellular metabolites required for generation of new biomass and to facilitate nutrient signaling. One of the earliest reports of a possible role for glucose metabolism in cancer proliferation was described by Otto Warburg in the early 1900s [5]. Warburg described a phenomenon in which cancer cells resort to the preferential use of glycolysis as a means of generating ATP even in the presence of oxygen. Normal cells on the contrary metabolize glucose to generate energy mainly through oxidative phosphorylation, and resort to the glycolytic pathway under anaerobic conditions [6-8]. The process of aerobic glycolysis (also known widely as the Warburg phenomenon) that occurs in nonhypoxic conditions also entails the generation of lactate [9]. This finding is surprising given that aerobic glycolysis is far less efficient than mitochondrial respiration and in recent years researchers have determined other reasons why cancer cells utilize this pathway. One of such reasons is that cell signaling pathways that are important in cancer cell growth are also indicated in the regulation of metabolic pathways [1,10]. The importance of this regulation of the metabolic pathways is that this facilitates the incorporation of nutrients essential for cell proliferation into biomass [1]. It has also been shown that activation of certain oncogenes, for example, $c-m y c$, ras and $s r c$, and transcription factors, such as hypoxia inducible factor-1a, can induce overexpression and the activity of glycolytic enzymes and GLUTs [11,12].

This property of increased glucose uptake in cancer cells is the basis of using $\left[{ }^{18} \mathrm{~F}\right]$ fluoro-2deoxyglucose (FDG) PET in the diagnosis and monitoring of some types of cancers. FDGPET is routinely used in the staging, evaluation of local disease and recurrence in malignancy [13]. Intracellular FDG concentration, which is measured via PET, directly correlates with glucose uptake [14]. Of note, FDG uptake is not limited to malignancy and can be seen in some benign tumors, and it has been suggested that this is as a result of microvascular density and vascular permeability instead of over-expression of GLUT [15]. Examples of benign tumors in which there can be increased FDG uptake include schwannomas and leiomyomas [15].

There are also recent studies in vivo that suggest that short-term starvation and, therefore, glucose restriction during chemotherapy promotes tumor retardation, potentiates chemotherapy and increases cancer-free survival in mice with breast cancer, melanoma and 
neuroblastoma [16 $\left.\boldsymbol{\square}^{\mathbf{m}}\right]$ due to reduced toxicity in normal tissues, underscoring the benefits of targeting glucose utilization for cancer therapy.

\section{THE GLUCOSE TRANSPORTER FAMILY}

The GLUTs' main function is to ensure that there is constant availability of glucose for metabolism, and they do this by controlling the movement of glucose between intracellular and extracellular compartments [2,17]. The genes of the GLUT family belong to the $2 \mathrm{~A}$ solute carrier family, and they have the gene symbol SLC2A. There are currently 14 isoforms of the GLUT genes described, and these isoforms can be grouped into three classes based on their primary sequences $[3,4,17]$. The first class of genes (class I) are known as the classical transporters and they are made up of GLUT 1-4 and GLUT 14, which is considered to be a gene duplicate of GLUT3. Class II is made up of GLUT5, 7, 9 and 11, whereas class III is composed of GLUT6, 8,10,12 and 13. GLUT 13 is also known as the $\mathrm{H}^{+}$ myoinositol transporter (HMIT) [3].

Although many isoforms of the GLUTs exist, they have similar transmembrane anatomy, with a large, highly conserved transmembrane domain and an asymmetric, less conserved cytoplasmic and exoplasmic domain [2]. They, however, differ in their functions in respect to subcellular localization, substrate recognition and transport kinetics [2,3]. The 14 isoforms have 14-63\% identical protein sequences among themselves with 30-79\% of them being conserved [17]. All the isoforms also have 12 transmembrane helices that are based on the original hydropathy plot for GLUT 1 . The different GLUTs have varied distribution in tissue and are usually cell-specific. They also have different affinities for glucose and other hexoses, such as fructose and galactose [2,3,17].

\section{GLUCOSE TRANSPORTER CLASS I}

This family of GLUTs was the first group to be described and is the most characterized. GLUT 1 and GLUT 4 are the most commonly studied [18]. In 1985, GLUT 1 was the first isoform to be cloned. GLUT 1 is expressed in erythrocytes, placenta, endothelial cells and, therefore, regulates entry across the blood-brain barrier; it has been found to be altered in conditions of cellular differentiation and transformation, in the presence of growth factors, insulin and stress [19]. Compared with the other GLUTs, GLUT1 has a high affinity for glucose and, therefore, plays a significant role in tissues that are highly dependent on glucose for energy [20]. GLUT 1 has been found to be overexpressed in a variety of both solid and hematological malignancies [19-21]. The variety of cancers in which GLUT1 has been over-expressed include but are not limited to diffuse large B-cell lymphoma, colorectal carcinomas, hepatocellular carcinoma, head and neck cancer, gastrointestinal stromal tumor (GIST), prostate carcinoma, thyroid carcinoma, renal cell cancer, lung cancer, pancreatic cancer, sarcomas and laryngeal carcinomas. In these cancers, there are data that are for and against the concept that the degree of GLUT 1 overexpression correlates with the grade of tumor, stage of cancer and clinical outcome [20,22-28].

GLUT 4 is found in tissues that are generally insulin-sensitive tissue such as cardiac, skeletal and muscular tissue. It has been studied widely in type II diabetes mellitus and obesity, and 
its role in tumori-genesis to a lesser extent [17]. The stimulation of GLUT 4 and its subsequent uptake of glucose in tissue has been linked to activation of the phosphatidylinositol 3-kinase-AKT pathway. Studies in our laboratory have demonstrated a key role for GLUT 4 in sustaining multiple myeloma cell viability and proliferation [29 $]$. It has also been shown that in GIST cells after treatment with imatinib, there is a decrease in GLUT 4 present in the plasma membrane [30]. This was thought to be as a result of endocytosis of GLUT 4 from the plasma membrane, or sequestration of intracellular microsomes, and was independent of expression of constitutively active AKT [30], also underscoring a role for GLUT4 in maintenance of these cancers.

GLUT 2 with a $\mathrm{K}_{m}$ of approximately $17 \mathrm{mmol} / 1[17,27]$ has the lowest affinity for glucose. It has been expressed in the cancer cell lines of the small intestine, kidney, breast, insulinoma, colon and pancreas. GLUT 2 and GLUT 5 are the transporters primarily indicated in fructose transport in humans, albeit GLUT 2 to a lesser extent than GLUT 5 $[2,17,22]$. GLUT 3 is another transporter in this class, which has been relatively well studied and has been found to be expressed predominantly in the brain. It is, however, also found in tissues that have high glucose demand, such as the placenta and testes [17]. Cancer cell lines in which GLUT 3 has been investigated include the breast, choriocarcinoma, ovarian, colorectal, retinoblastoma and rhabdomyosarcoma [2]. There have been conflicting reports of the role of tumor protein 53 (P53) in the regulation of GLUT 3; there have been reports that GLUT 3 expression is regulated via a p53-independent pathway, whereas another group suggests that the overexpression of p53 wild-type led to GLUT 3 suppression $[31,32]$.

\section{GLUCOSE TRANSPORTER CLASS II}

This group of GLUTs is sometimes referred to as the 'odd' GLUTs and a major characteristic of this class is their ability to transport fructose [2]. GLUT 2 and GLUT 5 were historically the transporters indicated in human fructose transport, more recently though the other GLUTs in this class role in fructose transport has been described [17,22].

GLUT 5 is found abundantly in the kidneys, testes, small intestine, muscle and adipose tissue. It is quite specific in its action of transporting fructose and lacks the ability to transport other hexose sugars [33- ]. GLUT 5 has been postulated to be important in tumorigenesis and has been found to be overexpressed in some types of cancer compared with normal cells, for example, renal cell carcinoma (RCC), breast and prostate cancer $[22,33 \cdot, 34 \square]$. The role of fructose in the form of 6-deoxy-6-[ $\left.{ }^{18} \mathrm{~F}\right]$ fluoro-D-fructose $\left(\left[{ }^{18} \mathrm{~F}\right]\right.$ (FDF) has been evaluated as an alternative PET radiotracer in cancers in which $\left[{ }^{18} \mathrm{~F}\right]$-FDGPET is not ideal, for example, breast cancer [34 - ]. GLUT7 was the last member of this family to be cloned, is expressed in the small intestine, colon and the prostate and testis to lesser extent. It has a high affinity for both glucose and fructose in humans, and its role in cancer metabolism is yet to be elucidated [17,35].

GLUT 9 is found primarily in the liver and kidney, but has also been found in the lung, leucocytes and the small intestine. There are variable reports on its ability to act as a glucose/fructose transporter; it recently was reported to be a high affinity transporter of urate [36] with implications in hyperuricemia. It has been postulated that its ability to act as a high 
capacity transporter of urate is in part due to enabling exchange with hexose sugars [17,36]. GLUT 11, the last transporter in this group, is primarily localized in the plasma membrane and can be found in a variety of tissue cells, including but not limited to the heart, placenta, kidney, skeletal muscle, adipose tissue and pancreas [17,29 $]$. To date, three isoforms have been described: GLUT 11A, GLUT 11B and GLUT 11C, and they are known to have a relatively low affinity for fructose and glucose compared with other GLUTs [17,29 $]$.

\section{GLUCOSE TRANSPORTER CLASS III}

On review of the literature, this group of GLUTs' role in cancer is less well characterized. GLUT 6 is found predominantly in the brain, spleen and white blood cell [17]. The only report of GLUT 6 overexpression in a malignancy was found in chronic lymphocytic leukemia that was associated with trisomy 12 , and this overexpression was determined not to be significant [37]. GLUT8 is routinely expressed in the testis, adrenal gland, liver, spleen and lung. Most recently, however, it has been reported to be overexpressed in multiple myeloma as well [17,29 ]. Recent studies from our laboratory suggest that GLUT 8 may play a role in intracellular glucose transport in the endoplasmic reticulum to potentially regulate protein glycosylation [29- ]. GLUT 12 is found to be localized in the perinuclear region and plasma membrane of prostate and breast cancer cell lines. It has also been expressed in heart, small intestine and skeletal muscle [3,17]. GLUT 10 and GLUT 13 (HMIT) are yet to be characterized in specific cancer cells/lines.

\section{TARGETING GLUTS AS A THERAPEUTIC INTERVENTION IN CANCER}

It has been observed that cancer cells are more susceptible to glucose deprivation compared with normal cells. Various studies have demonstrated that inhibition of glucose transport results in apoptosis and can also decrease cancer cell proliferation [38,39 $]$. Taking a step further, investigators have attempted to inhibit various steps in the glycolytic process so as to induce apoptosis. Examples of enzymes that have been targeted include pyruvate dehydrogenase, lactate dehydrogenase and hexokinase [38].

Although elevated expression levels of GLUT 1 are detected across numerous cancers, the actual contribution of this transporter can be determined only by specific ribonucleic acid interference approaches. GLUT1 is responsible for basal glucose transport in all cell types, and it has been shown that its level of expression correlates with the degree of invasion and metastatic potential of cancers $[38,39-$ ]. Treatment of various lung and breast cancer cell lines with anti-GLUT 1 antibodies was found to induce apoptosis. There have also been reports of synergistic effects after these drugs were combined with existing cancer chemotherapeutic agents [38]. Given the widespread expression patterns of GLUT 1 and its role in transport of glucose across the blood-brain barrier, it is preferable to target transporters with far more limited expression profiles.

\section{GLUT-SPECIFIC INHIBITORS}

STF-31 belongs to the second class in the group of compounds pyridyl anilino thiazoles, referred to as the 3 -series class. Its main mechanism of action is to inhibit glucose uptake 
and utilization. Chan et al.[26] investigated the effect of STF-31 in RCC, and they found that it was cytotoxic in RCC expressing GLUT 1 . They also found that RCC was rescued from the cytotoxic effects of STF-31 if they expressed high amounts of GLUT 2.

Genistein is a naturally occurring isoflavone compound (a derivative of soy) [40,41], which showed that it has a direct competitive inhibitory effect on GLUT 1, which results in inhibition of the transport of hexoses and dehydroascorbic acid across the plasma cell membrane [42]. Of note, genistein and other isoflavones have natural tyro-sine kinase inhibitory activities [43].

Fasentin is the death receptor stimuli and FAS-sensitizing compound that was described by Wood et al. [44]. Fasentin was found to bind directly to GLUT 1 and, thereby, inhibited glucose uptake of prostate cancer cells (PPC1) and U-937 leukemia cells, sensitizing these cells to FAS. Fasentin's inhibitory effect on the other GLUTs still needs to be elucidated.

Histone deacetylase inhibitors are a group of therapeutics that have recently been found to play a role in alteration of cancer cell metabolism. They were found to inhibit glucose uptake in multiple myeloma cells and acute promyelocytic leukemia cells, resulting in reduction of GLUT 1 expression leading to apoptosis [45].

Protease inhibitors, such as ritonavir and associated compounds, were first made famous for their use in the treatment of HIV/AIDS. It has been demonstrated that they have off-target inhibitory effects on GLUT 4, thereby decreasing glucose consumption in cancer [29 ${ }^{\mathbf{m}}$.

\section{CONCLUSION}

There is a recent resurgence of interest investigating glucose metabolism in cancer. A greater understanding of the reliance of cancers on individual GLUTs will likely lead to new targeting modalities for cancer therapy. In addition, establishing GLUTs as rate-limiting checkpoints across a broader spectrum of cancers, identifying alternate roles for GLUTs that are not high-affinity transporters and a role for compensatory metabolism in overcoming glucose-deprivation elicited stress are aspects that need to be considered in order to effectively target GLUTs for therapy. The preferred outcome would be to discover new therapeutic agents that are specific to the cancer cell's metabolism without causing untoward effect on the metabolism of the host.

\section{Acknowledgements}

S.T.R. has received funding from Cancer Center Support Grant (NCI CA060553).

\section{REFERENCES AND RECOMMENDED READING}

Papers of particular interest, published within the annual period of review, have been highlighted as:

- of special interest

- $\mathbf{a}$ of outstanding interest 
Additional references related to this topic can also be found in the Current World Literature section in this issue (p. 761).

1. Vander Heiden MG, Cantley LC, Thompson CB. Understanding the warburg effect: the metabolic requirements of cell proliferation. Science 2009; 324:1029-1033. [PubMed: 19460998]

2. Medina RA, Owen GI. Glucose transporters: expression, regulation and cancer. Biol Res 2002; 35:9-26. [PubMed: 12125211]

3. Macheda ML, Rogers S, Best JD. Molecular and cellular regulation of glucose transporter (glut) proteins in cancer. J Cell Physiol 2005; 202:654-662. [PubMed: 15389572]

4. Krzeslak A, Wojcik-Krowiranda K, Forma E, et al. Expression of glut1 and glut3 glucose transporters in endometrial and breast cancers. Pathol Oncol Res 2012; 18:721-728. [PubMed: 22270867]

5. Koppenol WH, Bounds PL, Dang CV. Otto Warburg's contributions to current concepts of cancer metabolism. Nat Rev Cancer 2011; 11:325-337. [PubMed: 21508971]

6. Shanmugam M, McBrayer SK, Qian J, et al. Targeting glucose consumption and autophagy in myeloma with the novel nucleoside analogue 8-aminoadenosine. J Biol Chem 2009; 284:2681626830. [PubMed: 19648108]

7. Shanmugam M, McBrayer SK, Rosen ST. Targeting the warburg effect in hematological malignancies: from PET to therapy. Curr Opin Oncol 2009; 21:531-536. [PubMed: 19587591]

8. Ganapathy V, Thangaraju M, Prasad PD. Nutrient transporters in cancer: relevance to Warburg hypothesis and beyond. Pharmacol Ther 2009; 121:29-40. [PubMed: 18992769]

9. Mathupala SP, Ko YH, Pedersen PL. The pivotal roles of mitochondria in cancer: Warburg and beyond and encouraging prospects for effective therapies. Biochim Biophys Acta 2010; 1797:12251230. [PubMed: 20381449]

10 . Vander Heiden MG. Targeting cancer metabolism: a therapeutic window opens. Nat Rev Drug Discov 2011; 10:671-684. [PubMed: 21878982] An up-to-date review of cancer metabolism, current and potential targeting options.

11. Rodriguez-Enriquez S, Marin-Hernandez A, Gallardo-Perez JC, Moreno-Sanchez R. Kinetics of transport and phosphorylation of glucose in cancer cells. J Cell Physiol 2009; 221:552-559. [PubMed: 19681047]

12. Marin-Hernandez A, Rodriguez-Enriquez S, Vital-Gonzalez PA, et al. Determining and understanding the control of glycolysis in fast-growth tumor cells. Flux control by an overexpressed but strongly product-inhibited hexokinase. FEBS J 2006; 273:1975-1988. [PubMed: $16640561]$

13. Kaida H, Ishibashi M, Yuzuriha M, et al. Glucose transporter expression of an esophageal gastrointestinal tumor detected by F-18 FDG PET/CT. Clin Nucl Med 2010; 35:505-509. [PubMed: 20548142]

14. Garcia Boy R, Knapp EM, Eisenhut M, et al. Enzymes/transporters. Handbook of experimental pharmacology 2008; 185 (Pt 2):131-143.

15. Hirose Y, Kaida H, Ishibashi M, et al. Glucose transporter expression of intraductal papilloma of the breast detected by fluorodeoxyglucose positron emission tomography. Jpn J Radiol 2011; 29:217-221. [PubMed: 21519997]

$16 \square$. Lee C, Raffaghello L, Brandhorst S, et al. Fasting cycles retard growth of tumors and sensitize a range of cancer cell types to chemotherapy. Sci TranslMed 2012; 4:124ra127.This study demonstrates the effect of fasting on tumor growth and response to chemotherapy.

17. Augustin R The protein family of glucose transport facilitators: it's not only about glucose after all. IUBMB Life 2010; 62:315-333. [PubMed: 20209635]

18. Frolova AI, Moley KH. Glucose transporters in the uterus: an analysis of tissue distribution and proposed physiological roles. Reproduction 2011; 142:211-220. [PubMed: 21642384]

19. Jun YJ, Jang SM, Han HL, et al. Clinicopathologic significance of GLUT1 expression and its correlation with apaf-1 in colorectal adenocarcinomas. World J Gastroenterol 2011; 17:18661873. [PubMed: 21528061]

20. Luo XM, Zhou SH, Fan J. Glucose transporter-1 as a new therapeutic target in laryngeal carcinoma. J Int Med Res 2010; 38:1885-1892. [PubMed: 21226991] 
21. Grabellus F, Nagarajah J, Bockisch A, et al. Glucose transporter 1 expression, tumor proliferation, and iodine/glucose uptake in thyroid cancer with emphasis on poorly differentiated thyroid carcinoma. Clin Nucl Med 2012; 37:121-127. [PubMed: 22228332]

22. Reinicke K, Sotomayor P, Cisterna P, et al. Cellular distribution of GLUT-1 and glut-5 in benign and malignant human prostate tissue. J Cell Biochem 2012; 113:553-562. [PubMed: 21938742]

23. Sakashita M, Aoyama N, Minami R, et al. glut1 expression in t1 and t 2 stage colorectal carcinomas: its relationship to clinicopathological features. Eur J Cancer 2001; 37:204-209. [PubMed: 11166147]

24. Fang J, Luo XM, Yao HT, et al. Expression of glucose transporter-1, hypoxiainducible factor-1alpha, phosphatidylinositol 3-kinase and protein kinase b (akt) in relation to [(18)f]fluorodeoxyglucose uptake in nasopharyngeal diffuse large b-cell lymphoma: a case report and literature review. J Int Med Res 2010; 38:2160-2168. [PubMed: 21227022]

25. Basturk O, Singh R, Kaygusuz E, et al. glut-1 expression in pancreatic neoplasia: implications in pathogenesis, diagnosis, and prognosis. Pancreas 2011; 40:187-192. [PubMed: 21206329]

26. Chan DA, Sutphin PD, Nguyen P, et al. Targeting glut 1 and the warburg effect in renal cell carcinoma by chemical synthetic lethality. Sci Transl Med 2011; 3:94ra70.

27. Amann T, Hellerbrand C. glut1 as a therapeutic target in hepatocellular carcinoma. Expert Opin Ther Targets 2009; 13:1411-1427. [PubMed: 19874261]

28. Carvalho KC, Cunha IW, Rocha RM, et al. glut1 expression in malignant tumors and its use as an immunodiagnostic marker. Clinics (Sao Paulo) 2011; 66:965-972. [PubMed: 21808860]

29 . McBrayer SK, Cheng JC, Singhal S, et al. Multiple myeloma exhibits novel dependence on glut4, glut8, and glut11: implications for glucose transporter-directed therapy. Blood 2012; 119:4686-4697. [PubMed: 22452979] This study illustrates myeloma dependence on glut 4 activity and proposes newly described functions for glut 8 and glut 11 in myeloma.

30. Tarn C, Skorobogatko YV, Taguchi T, et al. Therapeutic effect of imatinib in gastrointestinal stromal tumors: Akt signaling dependent and independent mechanisms. Cancer Res 2006; 66:5477-5486. [PubMed: 16707477]

31. Watanabe M, Naraba H, Sakyo T, Kitagawa T. DNA damage-induced modulation of glut3 expression is mediated through p53-independent extracellular signal-regulated kinase signaling in hela cells. Mol Cancer Res 2010; 8:1547-1557. [PubMed: 20870738]

32. Kawauchi K, Araki K, Tobiume K, Tanaka N. P53 regulates glucose metabolism through an ikk-nfkappab pathway and inhibits cell transformation. Nat Cell Biol 2008; 10:611-618. [PubMed: 18391940]

33 . Medina Villaamil V, Aparicio Gallego G, Valbuena Rubira L, et al. Fructose transporter glut5 expression in clear renal cell carcinoma. Oncol Rep 2011;25:315-323. [PubMed: 21165569] This study desribes a relationship between increased fructose uptake, GLUT 5 expression and progression of clear cell RCC.

34 - Wuest M, Trayner BJ, Grant TN, et al. Radiopharmacological evaluation of 6- deoxy-6[18f]fluoro-d-fructose as a radiotracer for pet imaging of glut5 in breast cancer. Nucl Med Biol 2011; 38:461-475. [PubMed: 21531283] This study describes a potential role for [18F]-FDF as radiotracer in cancers in which FDG-PET is not an option.

35. Cheeseman C glut7: a new intestinal facilitated hexose transporter. Am J Physiol Endocrinol Metab 2008; 295:E238-E241. [PubMed: 18477702]

36. Caulfield MJ, Munroe PB, O’Neill D, et al. Slc2a9 is a high-capacity urate transporter in humans. PLoS Med 2008; 5:e197. [PubMed: 18842065]

37. Porpaczy E, Bilban M, Heinze G, et al. Gene expression signature of chronic lymphocytic leukaemia with trisomy 12. Eur J Clin Invest 2009; 39:568-575. [PubMed: 19453646]

38. Zhang W, Liu Y, Chen X, Bergmeier SC. Novel inhibitors of basal glucose transport as potential anticancer agents. Bioorg Med Chem Lett 2010; 20:2191-2194. [PubMed: 20194024]

39 . Wang D, Chu PC, Yang CN, et al. Development of a novel class of glucose transporter inhibitors. J Med Chem 2012; 55:3827-3836. [PubMed: 22468970] This article describes a new class of GLUT inhibitors that are derived from a peroxisome proliferator-activated receptor-ginactive analog. 
40. Velentzis LS, Woodside JV, Cantwell MM, et al. Do phytoestrogens reduce the risk of breast cancer and breast cancer recurrence? What clinicians need to know. Eur J Cancer 2008; 44:17991806. [PubMed: 18614351]

41. Horn-Ross PL, John EM, Lee M, et al. Phytoestrogen consumption and breast cancer risk in a multiethnic population: the Bay Area Breast Cancer Study. Am J Epidemiol 2001; 154:434-441. [PubMed: 11532785]

42. Vera JC, Reyes AM, Carcamo JG, et al. Genistein is a natural inhibitor of hexose and dehydroascorbic acid transport through the glucose transporter, glut1. J Biol Chem 1996; 271:8719-8724. [PubMed: 8621505]

43. Vera JC, Reyes AM, Velasquez FV, et al. Direct inhibition of the hexose transporter glut1 by tyrosine kinase inhibitors. Biochemistry (Mosc) 2001; 40:777-790.

44. Wood TE, Dalili S, Simpson CD, et al. A novel inhibitor of glucose uptake sensitizes cells to fasinduced cell death. Mol Cancer Ther 2008; 7:3546-3555. [PubMed: 19001437]

45. Wardell SE, Ilkayeva OR, Wieman HL, et al. Glucose metabolism as a target of histone deacetylase inhibitors. Mol Endocrinol 2009; 23:388-401. [PubMed: 19106193] 


\section{KEY POINTS}

- A myriad of cancers exhibit increased glucose uptake and aerobic glycolysis, that is, the Warburg effect.

- Glucose transporters are rate-limiting checkpoints, abnormally regulated in cancer.

- Glucose transporters can be targeted in a relatively tumor cell-specific manner to block glucose-regulated processes more comprehensively for cancer therapy. 